\title{
Le sexe des oiseaux
}

\section{Simone Gilgenkrantz}

> Au cours de l'évolution, la séparation des individus en deux genres, mâle et femelle, s'est très tôt imposée. Puis, les facteurs masculinisants ou féminisants ont été peu à peu isolés des autres gènes. Ainsi sont apparus les gonosomes, chromosomes sexuels porteurs principalement - sinon essentiellement - de ces facteurs de différenciation vers l'un ou l'autre genre. D'une manière schématique, chez les vertébrés, l'évolution semble s'être faite dans deux directions opposées: tandis que, chez les mammifères, le système XX:XY détermine, par les facteurs portés par I'Y, la différenciation mâle, chez les oiseaux, en revanche, le système $Z Z / Z W$ entraîne, grâce au chromosome $W$, la différenciation femelle. $\varepsilon$ n réalité, la comparaison entre les deux systèmes se révèle infiniment plus complexe. Chez les oiseaux, comme chez les vertébrés inférieurs, la différenciation gonadique est en grande partie conditionnée par les œstrogènes; certains gènes essentiels à la détermination sexuelle chez les mammifères sont exprimés également chez le poulet, mais leur profil d'expression diffère, démontrant leur plasticité fonctionnelle. Enfin, et surtout, on connaît encore mal les gènes de différenciation sexuelle portés par le chromosome W. Mais les études comparatives sur les voies suivies par les vertébrés, avec leurs divergences et leurs convergences, élargissent notre connaissance et notre compréhension des divers mécanismes de différenciation sexuelle. <

La sexualisation, un des facteurs importants de l'évolution des espèces vivantes, revêt de multiples facettes et ne se limite pas au système XX:XY des mammifères. Chez les oiseaux, la détermination sexuelle repose sur le système ZZ:ZW qui est, dans une certaine mesure, diamétralement opposé : le chromosome $W$ est porté par

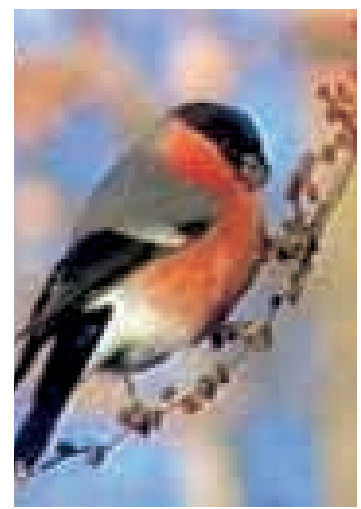

9 , rue Basse, 54330 Clerey-sur-Brenon, France. simone.gilgenkrantz@ les femelles, ce sont club-internet.fr donc elles qui sont hétérogamétiques tandis que les mâles sont homogamétiques. Mais l'analyse détaillée des mécanismes de la différenciation sexuelle aviaire, dans ses aspects génétiques et morphologiques, révèle des phénomènes complexes: (1) comme chez les vertébrés inférieurs, les œstrogènes jouent un rôle important; (2) les profils d'expression des gènes communs aux mammifères et aux oiseaux ne sont pas les mêmes dans les deux lignées; (3) des gènes récemment découverts interviennent spécifiquement dans la différenciation sexuelle aviaire. Cependant, paradoxalement, on n'a pas trouvé jusqu'à présent de gène déterminant la différenciation ovarienne sur le chromosome $W$ des oiseaux femelles, analogue du gène SRY intervenant dans la différenciation testiculaire chez les mammifères. Mais comme le souligne une excellente revue australienne toute récente [1], les données actuelles sur les mécanismes de la différenciation sexuelle des oiseaux offrent d'intéressantes perspectives sur l'ensemble des processus de sexualisation advenus au cours de l'évolution des vertébrés.

\section{Les gonosomes aviaires $Z$ et $\mathrm{W}$}

La lignée des oiseaux et celle des mammifères a divergé à partir d'ancêtres reptiliens il y a environ 350 millions d'années. Les oiseaux proviennent de reptiles diapsides ( 2 fosses temporales) et les mammifères de reptiles synapsides (une seule fosse temporale) (Figure 1). On connaît le divorce programmé depuis 250 millions d'années entre les chromosomes $X$ et $Y$. Ce dernier a progressivement réduit sa taille et, surtout, le nombre de ses 
gènes pour ne plus garder qu'une petite région pseudo-autosomique qui s'apparie à l'X au cours de la méiose masculine. De même, le chromosome W ( $1 \%$ du génome haploïde aviaire) ne possède plus qu'une petite région euchromatique à la partie terminale de son bras court. II forme un complexe synaptonémal avec le chromosome $Z$ pendant la méiose femelle. Mais les gonosomes aviaires ne procèdent pas des mêmes proto-gonosomes que $X$ et $Y$ puisque $Z$ est en partie synténique avec le chromosome 9 humain tout en contenant quelques blocs homologues en synténie avec des régions des chromosomes humains 5, 8 et 18 [2].

\section{Rôles des gonosomes : l'introuvable gène féminisant}

Contrairement à l'espèce humaine où les conséquences de la perte (45, $X$ : syndrome de Turner) ou de l'excès d'un gonosome (XXY : syndrome de Klinefelter) ont été bien étudiées et ont permis d'affirmer que la présence d'un y (avec SRY) est nécessaire et suffisante pour la différenciation testiculaire, aucune dysgonosomie aviaire permettant de déterminer le rôle de chacun des gonosomes n'a été observée. Certaines observations anciennes d'oiseaux latéralisés (mâle d'un côté : à droite, femelle de l'autre : à gauche) n'ont pas été confirmées par des études cytologiques ou moléculaires. Récemment, toutefois, un passereau gynandromorphe a été observé avec une latéralisation ZW femelle du côté gauche et ZZ mâle du côté droit [3]. En l'absence de cas d'aneuploïdie, l'hypothèse d'un effet létal - au stade embryonnaire - de la perte d'un gonosome a été évoquée. Il existe toutefois des poulets triploïdes. Dans ce cas, les animaux ont d'abord un état intersexuel, avec un testicule normal à droite et, à gauche, transitoirement, un ovotestis (mélange de tissus testiculaire et ovarien). Le phénotype est d'abord femelle puis les composants ovariens de l'ovotestis

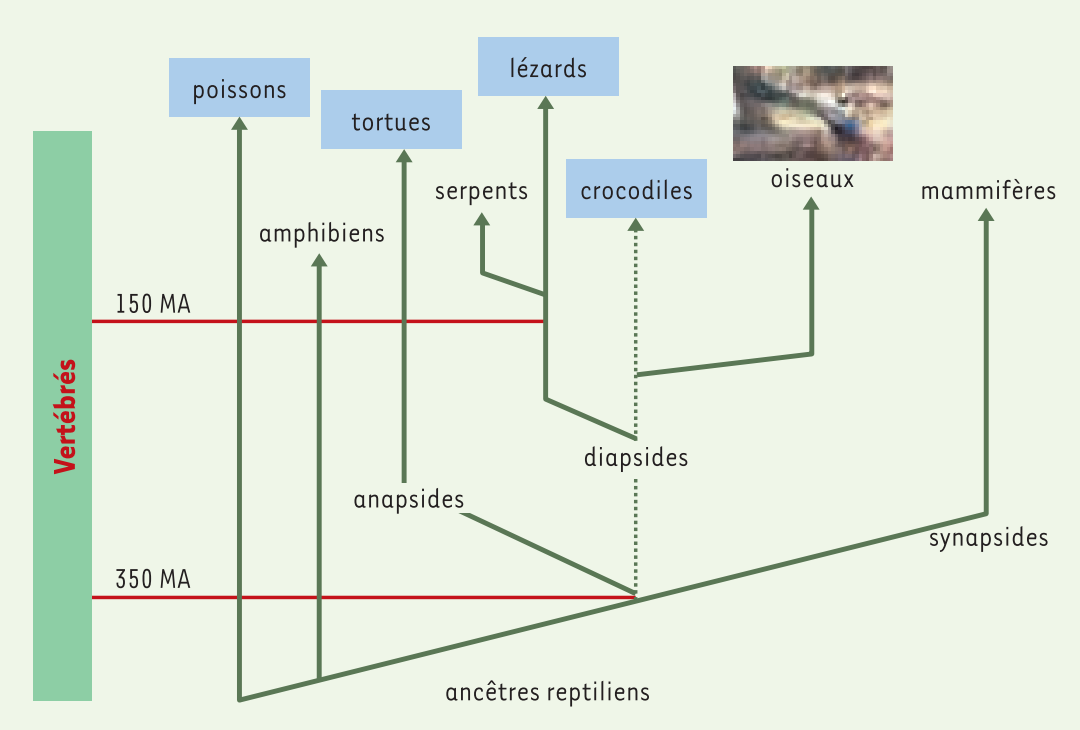

Figure 1. Évolution des vertébrés. Les reptiles diapsides auraient évolué vers les archosauriens (crocodiles/oiseaux) et vers les épidosauriens (lézards/serpents). Le fond bleu correspond aux animaux dont la détermination sexuelle est dépendante de la température. MA: millions d'années (d'après [1]). dégénèrent et, à la période de maturité sexuelle, les oiseaux deviennent phénotypiquement des coqs. Il semble donc que le $W$ porte un ou des déterminants femelles. Si c'est le cas, ils ne sont pas dominants puisque, chez les triploïdes ZZW, la régression du tissu ovarien s'effectue (à gauche) probablement sous l'effet des facteurs masculinisants portés en double dose par les deux chromosomes $Z$. Les gènes intervenant dans l'ovogenèse ne sont pas non plus portés par le chromosome W. En effet, si on injecte des cellules germinales ZZ à des embryons femelles ZW, certaines de ces cellules se retrouvent dans le tissu ovarien et sont capables d'accomplir une méiose pour devenir des ovocytes fonctionnels, alors qu'elles sont dépourvues de chromosome W. La méiose est donc celluleautonome et contrôlée par des gènes situés soit sur un autosome, soit sur le chromosome $Z$.

\section{Différenciation gonadique : l'étonnante lévo-féminisation}

On sait que, chez les mammifères, les gonades primitives se développent à partir du mésonéphros avec une couche superficielle, corticale, et une couche profonde, médullaire. La différenciation est plus rapide chez le mâle, avec involution de la région corticale et développement de la région médullaire qui héberge les cellules primordiales primitives. Chez la femelle, à l'inverse, c'est la région corticale qui se développe et héberge un stock non renouvelable de cellules germinales. S'il existe un décalage chronologique entre les sexes, le processus se déroule en tout cas de façon symétrique. En revanche, chez les oiseaux, la différenciation gonadique des femelles a une particularité : elle est asymétrique. Tandis qu'un ovaire se développe à gauche (avec épaississement de la région corticale comme chez les femelles de mammifères), la gonade droite, après un développement discret, régresse (Figure 2).

\section{L'importance des hormones}

Chez les mammifères euthériens, la gonadogenèse est insensible aux hormones exogènes. Chez les oiseaux, au contraire, l'adjonction d'œstrogènes peut provoquer une inversion sexuelle. Chez les femelles, l'aromatase - enzyme 
inhibitrice de la synthèse d'œstrogènes provoque une inversion sexuelle femelle $\rightarrow$ mâle qui est définitive. Quant aux mâles traités à l'œstradiol, ils se féminisent mais de façon réversible. Les deux enzymes nécessaires à la synthèse d'œstradiol, P-450 aromatase et $17 \beta \mathrm{HSD}$, ne sont exprimées que dans les gonades femelles au début de la différenciation gonadique $(J 6,5)$. Il est possible qu'un gène de détermination sexuelle porté par le $W$ active ces deux enzymes. En revanche, les androgènes n'ont pas d'action car le récepteur des androgènes est exprimé tardivement.

Pour que l'œstradiol puisse agir, son récepteur ( $\varepsilon R \alpha)$ doit être présent. Effectivement, il est exprimé dans les gonades des deux sexes avant la différenciation, dans la région corticale principalement. Chez les mâles, l'expression décroît par la suite. Chez les femelles, elle s'éteint dans la gonades droite. Le mécanisme contrôlant cette asymétrie est encore inconnu. Mais on peut dire que l'œstradiol synthétisé dans la zone médullaire induit la différenciation en ovaire en stimulant le cortex par l'intermédiaire de $\varepsilon R \alpha$. L'expression transitoire de ce récepteur chez les mâles $Z Z$ explique leur sensibilité aux œstrogènes exogènes, conduisant à une féminisation.

Si on greffe des testicules embryonnaires à des embryons femelles $Z W$ avant la différenciation gonadique, les deux gonades, droite et gauche, évoluent en oiseaux, la différenciation gonadique peut se faire en l'absence de cellules germinales. Ou bien, autre hypothèse, elle pourrait réprimer l'expression de l'aromatase chez les mâles. Mais ceci nécessiterait un dosage génique bien précis puisque l'AMH est aussi exprimée chez les femelles où l'expression de l'aromatase ne doit pas se produire. Peutêtre enfin, cette inversion de la différenciation gonadique est causée par un autre facteur, encore inconnu, sécrété par la greffe testiculaire.

\section{La cascade des gènes conservés chez les oiseaux}

Les gènes intervenant dans la différenciation sexuelle chez les mammifères sont de mieux en mieux connus. Les plus précoces dans la cascade sont des facteurs de transcription comme SFl (steroidogenic factor-1), WTl (Wilm's tumour-associated protein) et LHX9 (LIM homeobox protein). Ils sont remarquablement conservés chez les oiseaux et exprimés dans les gonades indifférenciées des deux sexes. Chez les mammifères, SFI et WTI activent l'expression de SRY. Ce produit étant absent chez les oiseaux, il importait de rechercher les rôles de ces facteurs dans les espèces aviaires. SFI agit en fait sur l'aromatase (qui est pourvu d'un site de liaison dans sa région promotrice). L'expression élevée de SFI dans les gonades femelles doit correspondre à la synthèse d'œstrogènes. Ensuite, elle décroît alors qu'elle se maintient chez les mâles. Ce même profil d'expression se retrouve chez les crocodiles qui font partie de l'espèce vivante la plus proches des oiseaux aujourd'hui.

Le SOX9 a aussi une expression différente selon le sexe chez tous les vertébrés. testicules [4]. Cette transformation se fait sous l'influence de l'hormone antimullérienne (AMH) produite par les cellules de Sertoli des testicules greffés. Chez les mammifères, I'AMH est exprimée plus tard dans la cascade des gènes intervenant dans la différenciation gonadique, bien après des gènes clés, comme SOX9 par exemple. Chez les oiseaux, l'expression d'AMH débute dans les deux sexes avant la différenciation gonadique. Mais son niveau d'expression doit être différent, nettement plus élevé chez les mâles que chez les femelles puisque son augmentation (en cas de greffe de testicule) entraîne une inversion du sexe.

Peut-être I'AMH joue-t-elle un rôle déterminant puisqu'elle apparaît juste avant la différenciation histologique des gonades. Elle pourrait empêcher la colonisation par les cellules germinales, nécessaire à la formation des ovaires chez les mammifères. Mais, chez les

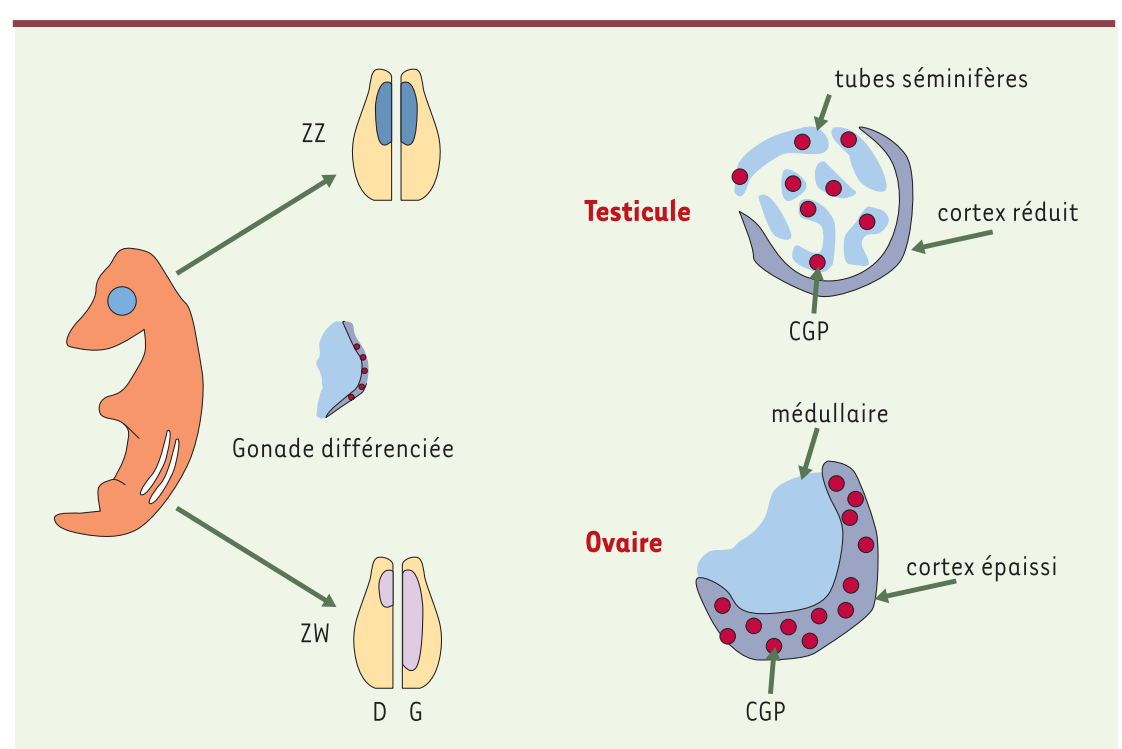

Figure 2. Différenciation gonadique chez le poulet. À 33,5 , les gonades primitives sont apparentes avec une zone corticale et une zone médullaire. Les cellules germinales primordiales se localisent d'abord dans la région corticale. La différenciation morphologique s'accomplit entre $J 5,5$ et J6,5. Chez le mâle, les cordons médullaires se développent en tubes séminifères contenant les cellules germinales. Chez les femelles, la région corticale s'épaissit, mais seule la gonade gauche se développe en testicule, tandis que la gonade droite régresse (d'après [1]). 
II est structurellement conservé chez les mammifères, les oiseaux et les reptiles. Mais alors que chez les mammifères, il interagit avec SFI et WTI pour activer l'AMH, il n'apparaît chez les oiseaux qu'après I'AMH, comme chez les crocodiles.

\section{À la recherche du facteur de détermination sexuelle}

Contrairement à ce qui se passe chez les mammifères pour le deuxième $X$, le deuxième chromosome $Z$ n'est pas inactivé chez les mâles. De nombreux gènes liés au Z sont transcrits en double. Le gène DMRTI - un bon candidat pour la détermination mâle - est absent sur le $W$ et n'est pas réglé par un effet de dosage génique. Il est exprimé en quantité beaucoup plus importante chez les mâles avant et pendant la différenciation gonadique. La protéine DMRTl est d'abord localisée dans les noyaux des cellules des cordons médullaires. Il est étonnant de voir que, chez la drosophile, ou chez Caenorhabditis elegans, les homologues de DMRTI ont aussi des fonctions spécifiques du mâle. DMRTI représente donc un très ancien gène de détermination mâle présent dans différents phylums. Chez les mammifères, la différence de dosage entre les deux sexes ne se manifeste qu'après le début de la différenciation gonadique. Chez la souris, l'invalidation ciblée de Dmrtl n'entraîne pas d'inversion sexuelle mais seulement un dysfonctionnement testiculaire post-natal. Tout se passe donc comme si DMRTl, déterminant mâle chez les vertébrés inférieurs et chez les oiseaux, avait été supplanté par SRY chez les vivipares.

L'hypothèse d'une interaction entre $W$ et $Z$ a été proposée dans un nouveau modèle de détermination sexuelle aviaire. Une équipe japonaise a en effet mis en évidence une région hyperméthylée et inactive sur le bras court des deux $Z$ chez les mâles (MHM) [5]. Dans l'unique chromosome $Z$ chez les femelles, cette région MHM est en revanche hypométhylée, transcrite avec un ARN non codant de haut poids molécu-

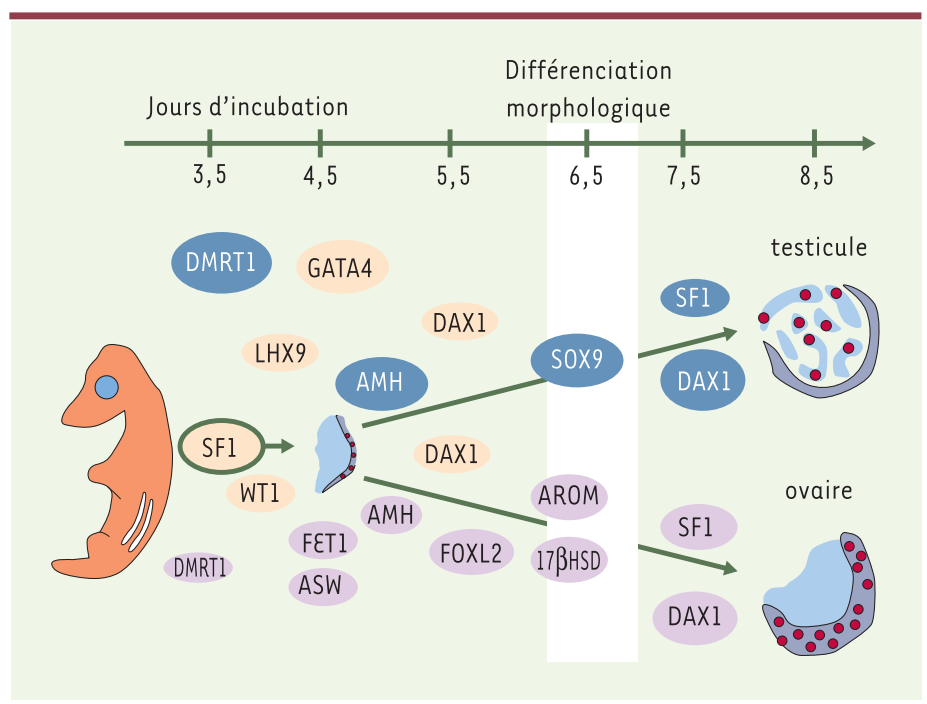

Figure 3. Expression des gènes durant la période de différenciation gonadique. Les cercles indiquent le début de l'expression, en bleu chez le mâle et en violet chez la femelle. La taille indique l'importance comparative de l'expression (d'après [1]). laire. Or, l'étude des animaux triploïdes est éclairante: alors que dans les gonosomes des triploïdes ZZZ, MHM est hyperméthylée et inactive sur les trois $Z$, chez les triploïdes $Z Z W$, la région MHM des deux $Z$ est hypométhylée et transcrite, comme si le chromosome $W$ intervenait à distance dans le statut de méthylation de la région MHM des Z. On est tenté de faire le parallèle avec le gène Xist des mammifères capable par accumulation d'ARN non codant d'entrấner une extension de la méthylation. En présence d'un $W$, la région MHM hypométhylée, en produisant un ARN qui s'accumule au site de transcription, provoque la répression de certains gènes avoisinants, en particulier de DMRTI situé juste sous la région MHM. S'il y a répression de DMRTI chez les femelles, elle ne doit pas être totale : bien qu'à faible dose, DMRTl est exprimé dans les gonades ZW (Figure 3).

\section{Les nouveaux gènes candidats}

Deux gènes ont été découverts au cours de ces dernières années. Le premier, ASW (avian sex W-linked) code pour une protéine qui a quelque homologie avec un inhibiteur de la protéine kinase $C$, d'où son autre nom : WPKCl. $A S W / W P K C 1$ se retrouve une quarantaine de fois sur le chromosome W, alors qu'il n'existe qu'une seule copie de l'homologue ZPKCI sur le chromosome Z. Sa présence en multiples exemplaires peut expliquer l'amplification de son signal. Il est en effet fortement exprimé chez les femelles, en particulier dans les gonades. Mais il n'est pas capable d'induire une véritable différenciation femelle chez les oiseaux ZZW. Ceux-ci, comme nous l'avons dit, ne développent à gauche, et transitoirement, qu'un ovotestis. De plus: (1) ASW/WPKCI n'est pas spécifique de tissu dans les embryons femelles; et (2) on ne le retrouve pas chez les autruches femelles qui ont pourtant une différenciation sexuelle analogue aux autres oiseaux. Mais il faut reconnaître que le groupe des ratites (autruches, émeus, casoars) a divergé très tôt au cours de l'évolution aviaire et qu'il possède encore des chromosomes sexuels homomorphiques.

Le second gène, récemment identifié, est un candidat à la détermination ovarienne beaucoup plus séduisant : FETI (female expressed transcrit) est localisé dans la région euchromatique du bras court du W. II n'a pas d'homologue sur le $Z$; il est exprimé presque exclusivement dans le système urogénital femelle, avec expression élevée pendant la période de différenciation (J4, 5 à J6,5). Son expression est asymétrique, beaucoup plus élevée dans la gonade gauche. II code pour une protéine prédite qui aurait une séquence signal et un domaine 
transmembranaire. Aucun orthologue d'ASW/WPKCI ni de FETI n'a été retrouvé chez les mammifères. Ces gènes (ou l'un d'entre eux) sont-ils les déterminants ovariens dans les lignages reptiles et oiseaux, analogues au SRY des mammifères ? Ce n'est pas impossible, mais il reste encore dans ce cas à trouver les gènes en aval, qui agiraient sur l'aromatase, car ASW/WPKCl et FETl interviennent beaucoup trop tôt pour agir directement sur cette enzyme (Figure 4).

Par ailleurs, comment vérifier que DMRTI sur le $Z$ ainsi que ASW/WPKCl et FETI sur le $W$ sont les bons candidats ? L'utilisation d'animaux transgéniques est trop longue et trop difficile compte tenu du mode de reproduction des oiseaux. Les stratégies les plus prometteuses devraient être, entre autres, I'utilisation de vecteurs rétroviraux pour une surexpression in ovo, ou bien l'invalidation de l'expression de gènes par des méthodes antisens.

\section{Conclusions}

L'étude des oiseaux, on le voit, fournit un excellent moyen d'analyse de l'évolution de la détermination sexuelle des vertébrés. Les différences entre le modèle des mammifères et celui des oiseaux élargissent notre vision de cette évolution. Situés à une étape intermédiaire, les oiseaux conservent encore certains processus existant chez les vertébrés inférieurs, comme le rôle clé des œstrogènes, mais ils possèdent aussi la différenciation gonadique et des gènes en commun avec les mammifères. Le dimorphisme sexuel de l'expression des gènes au niveau moléculaire est très précoce $(J 3,5)$ et précède le début de la différenciation histologique $(J 6,5)$. Une interaction de $W$ sur la région $M H M$ de $Z$, si elle se confirme, est intéressante : elle laisse entrevoir une stratégie interchromosomique qui n'a jamais été observée (jusqu'à présent) chez les mammifères. Enfin, l'asymétrie de développement gonadique des femelles, phénomène purement aviaire, rejoint étrangement d'anciennes croyances et des us et coutumes (semence femelle dans le testicule gauche selon Hippocrate; côté épître (gauche) pour les femmes dans les églises; place des boutons sur le côté gauche des vêtements féminins...) qui perdurent encore de nos jours et qui associent toujours féminité et côté gauche. $\diamond$

\section{SUMMARY}

\section{Bird sex determination}

During the evolution, sex determination occured early. Sex determining factors were progressively isolated from other genes in sexual chromosomes, or gonosomes. Among vertebrates, evolution took two opposite pathways : in mammals, the system of $X X: X Y$ sex determination, with $Y$ chromosome, in-duces male differenciation. In contrast, in birds, the system ZZ:ZW, with the $\mathrm{W}$ chromosome, induces female differenciation. But comparative studies show that the two pathways are not so simple. In the chicken as in the lower vertebrates, estrogens play a central role in gonadal sex differenciation. Several genes, show to be critical for mammalian determination, are also expressed in the chicken but their expression pattern differs, indicating functional plasticity. The W-linked female determinants remains still unknown. But comparative studies of the two pathways, with conserved and divergent elements, are broadening our understanding of sex determination. $\diamond$

\section{RÉFÉRENCES}

1. Smith CA, Sinclair AH. Sex determination : insights from the chicken. Bioessays $2004 ; 26$ : $120-32$.

2. Graves JAM, Shetty S. Sex from $W$ to $Z$ : evolution of vertebrate sex chromosomes and sex determining genes. J Exp Zool 2001 ; 2990 : 449-62.

3. Agate RJ, Grisham W, Wade J, et al. Neural, not gonadal, origin of brain sex differences, in a gynandromorphic finch. Proc Natl Acad Sci USA $2003 ; 100: 4873-8$.

4. Maraud R, Vergnaud 0 , Rashedi M. New insights on the mecanism of testis differentiation from the morphogenesis of experimentally induced testes in genetically female chicken embryos. Am J Anat 1990 ; $188: 429-37$.

5. Teranishi M, Shimada Y, Hori T, et al. Transcripts of the MHM region on the chicken $\mathrm{Z}$ chromosome accumulate as non-coding RNA in the nucleus of female cells adjacent to the DMRT1 locus. Chrom Res 2001 ; $9: 147-65$.

6. Smith CA, Smith MJ, Sinclair AH. Expression of chicken steroidogenis factor-1 during gonadal differentiation. Gen Comp Endocrinol 1999 ; $13: 445-54$.

7. Western DS, Harry JL, Graves JAM, Sinclair AH. Temperature-dependant sex determination in the American alligator : expression of SFI, WT1 and DAXI during gonadogenesis. Gene $2000 ; 241: 223-32$.
Figure 4. Les gonosomes aviaires $Z$ et $W$. A. Quelques gènes portés par les gonosomes aviaires. B. Mécanisme proposé pour la régulation de la zone MHM chez les femelles, par action d'un facteur porté par W (d'après [1]).
TIRÉS À PART

S. Gilgenkrantz 